\title{
Les néologismes en -age en français contemporain : héritage verbal et polysémie
}

\author{
Richard Huyghe* (iD) et Alizée Lombard (D) \\ Université de Fribourg \\ ${ }^{*}$ Corresponding author. Email: richard.huyghe@unifr.ch
}

(Received 31 March 2020; revised 09 November 2020; accepted 14 November 2020; first published online 16 November 2021)

\section{RÉSUMÉ}

Cet article porte sur les propriétés sémantiques et syntaxiques des néologismes déverbaux en -age en français contemporain. À partir de l'analyse d'un échantillon de 139 néologismes et de leurs bases verbales, selon le type de procès décrit, l'aspect lexical, la structure argumentale et la grille thématique, l'étude montre que la suffixation en -age produit essentiellement des noms de procès similaires à ceux décrits par leur base. L'hypothèse est formulée que les décalages aspectuels observés dans le lexique entre verbes et nominalisations en -age sont dus au processus de lexicalisation, et non à la construction morphologique. S'agissant de la sélection des bases, contrairement à ce qui est parfois défendu pour les noms lexicalisés, aucune prédilection pour les verbes transitifs n'apparaît, et la classe aspectuelle la plus représentée est celle des activités. Par ailleurs, la polysémie des néologismes en -age relève principalement du type 'action' / 'résultat', et certains éléments laissent penser qu'elle résulte d'une figure métonymique, et non de la dérivation. Enfin, il apparaît que la formation de noms en -age dans le discours peut être diversement motivée, et que de nombreux néologismes sont créés en surabondance lexicale.

\section{INTRODUCTION ${ }^{1}$}

La suffixation en -age, qui permet en français de construire des noms à partir de bases verbales, est au cour de plusieurs études récentes sur la concurrence et la polysémie affixales. L'intérêt pour -age tient au fait qu'il est l'un des suffixes de nominalisation les plus productifs en français. Nous souhaitons ici contribuer à l'étude de ce suffixe en nous focalisant sur les néologismes qu'il permet de former. Ce choix est motivé par le fait que les néologismes permettent d'appréhender les procédés dérivationnels dans ce qu'ils ont de plus régulier synchroniquement, indépendamment de l'opacification

\footnotetext{
${ }^{1}$ Nous remercions les trois relecteurs ou relectrices anonymes pour leurs commentaires et suggestions, qui nous ont permis d'améliorer la qualité de cet article, ainsi que Clément Plancq pour son aide dans l'extraction automatique des données.
} unrestricted re-use, distribution, and reproduction in any medium, provided the original work is properly cited. 
sémantique qui peut accompagner la lexicalisation. À cet égard, notre travail s'inspire de ceux de Plag et al. (2018), qui étudient les néologismes déverbaux en -ment en anglais, et de Dal et al. (2018), qui comparent en français les noms en -age et en -ment rencontrés sur le web et présents dans les dictionnaires.

L'étude que nous présentons porte spécifiquement sur les propriétés sémantiques et syntaxiques des noms suffixés en -age (désormais $\mathrm{N}$-age), et sur leur construction à partir des bases sélectionnées. Ce travail est exploratoire, et constitue une première étape dans l'analyse comparative de néologismes créés selon différents procédés morphologiques en français. Nous nous interrogeons à ce stade sur deux éléments fondamentaux : l'héritage des propriétés des bases verbales et la polysémie des $\mathrm{N}$-age. D'une part, nous souhaiterions savoir si les $\mathrm{N}$-age, lorsqu'ils dénotent des procès, préservent l'aspect lexical, la structure argumentale, la grille thématique de leur base ou, lorsqu'ils dénotent des entités, renvoient à un élément inclus dans la structure sémantique du verbe. D'autre part, nous nous demandons quelles sont les différentes configurations d'acceptions possibles des $\mathrm{N}$-age à partir d'une base donnée, et si la polysémie des déverbaux en -age doit être considérée comme le fait de la construction morphologique ou d'une figure de sémantique lexicale (métaphore ou métonymie). De manière annexe, on peut s'interroger sur les raisons de l'apparition des néologismes en -age, et notamment se demander si les $\mathrm{N}$-age créés en discours sont destinés à combler un vide lexical, ou s'ils constituent des cas de surabondance formelle.

L'article est organisé comme suit. Dans la première section, nous présentons succinctement les travaux existants sur le suffixe -age en français, portant sur la concurrence avec les procédés morphologiques proches et la polysémie du suffixe. La deuxième section décrit la méthodologie d'extraction et d'annotation des données. Dans la troisième section, nous rendons compte de l'analyse de ces données, et discutons différents points théoriques concernant l'héritage et la polysémie des $\mathrm{N}$-age. Trois hypothèses principales seront défendues. Primo, les $\mathrm{N}$-age néologiques ne privilégient pas la sélection de bases verbales transitives, et la classe aspectuelle la plus représentée parmi les bases est celle des activités. Secundo, contrairement à ce qu'on observe pour les N-age lexicalisés, il n'y a pas de décalage aspectuel entre les bases verbales et les $\mathrm{N}$-age néologiques dénotant des procès. Tertio, la polysémie des néologismes en -age relève essentiellement du type 'action' / 'résultat', certains éléments suggérant qu'elle résulte d'une métonymie, et non d'une construction morphologique.

\section{Le suffixe -age en question}

Il existe au moins deux suffixes -age en français. Le premier sélectionne des bases nominales et construit des noms collectifs, comme branchage, feuillage, rayonnage. Le second sélectionne des bases verbales et construit des noms dont la dénotation est liée au procès verbal, comme dérapage, lavage, tissage. La distinction entre les deux constructions est généralement claire. Il existe des cas d'ambiguité morphosémantique, théoriquement non problématiques, dans lesquels la double analysabilité du nom correspond à deux acceptions distinctes d'un même vocable (ex. grillage 'ensemble de grilles' vs 'action de griller'). Plus délicats sont les cas de sous-détermination constructionnelle, i.e. les cas de noms doublement 
analysables dans une acception donnée, tel balisage, qui dans son sens d'objet peut être défini comme 'ensemble de balises' ou comme 'résultat de l'action de baliser'. Par ailleurs, tous les noms qui se terminent par -age et dans lesquels on pourrait voir une base nominale ne sont pas des noms collectifs (ex. coquillage, paysage, alpage, $\mathrm{cf}$. Berrendonner et Clavier, 1997), et tous les noms de procès en -age ne sont pas déverbaux - nous trouvons dans le corpus $\mathrm{frWaC}$ des néologismes comme pipelettage, mais pas de verbe pipeletter, si bien que l'analyse morphologique du $\mathrm{N}$-age selon l'un des patrons de construction établis est incertaine. Nous nous en tiendrons dans cette étude aux noms relevant clairement de la construction déverbale. Cette dernière est la plus étudiée à ce jour.

\subsection{Concurrence dérivationnelle}

La construction déverbale en -age est souvent appréhendée dans des situations de concurrence morphologique. ${ }^{2}$ La suffixation en -age est ainsi comparée avec celles en -ment et en -ion (Dubois, 1962 ; Lüdtke, 1978 ; Kelling, 2001 ; Martin, 2010 ; Uth, 2010 ; Dal et al., 2018 ; Wauquier et al., 2018 ; Fradin, 2019), avec la conversion en -ée (Ferret et al., 2010 ; Ferret et Villoing, 2012), ainsi qu'avec la conversion de verbe à nom en général (Missud, 2019). Même s'il est admis que les $\mathrm{N}$-age peuvent dans certains cas dénoter des objets, la concurrence examinée porte le plus souvent sur les noms qui décrivent des " éventualités ", i.e. toutes formes de situations, qu'elles soient statiques ou dynamiques (Bach, 1986 ; Asher, 1993 ; Filip, 1999 ; inter alia). Le postulat classique est que des procédés de construction différents se voient assigner des fonctions distinctes, et les études tentent de mettre en évidence des différences (fussent-elles tendancielles) entre -age et les autres formations. On recherche, d'une part, des différences dans la sélection des bases. Ainsi, selon Dubois (1962) et Lüdtke (1978), -age se distingue de -ment par sa prédilection pour les bases transitives ( $v s$ intransitives pour -ment). Kelling (2001) estime que le principe fondamental de distinction entre -age et -ment n'est pas la transitivité du verbe de base, mais le degré de protoagentivité de son sujet, -age sélectionnant des bases plus protoagentives que -ment. Le critère de la transitivité est toutefois repris par Ferret et al. (2010) dans leur étude de la concurrence entre -age et -ée, avec l'idée que -age sélectionne plutôt des bases transitives ( $v s$ inaccusatives pour -ée). L'hypothèse d'une sélection aspectuelle de la base est examinée par Martin (2010), qui montre que l'(a)télicité du verbe de base n'a pas d'influence sur le choix entre

\footnotetext{
${ }^{2}$ La notion de concurrence morphologique soulève d'importantes questions (cf. Gardani et al., 2019 pour une présentation générale), et on peut s'interroger sur sa définition exacte. L'identité syntaxique et/ou sémantique des bases, et l'identité syntaxique et/ou sémantique des mots construits sont-elles requises ? Comment définit-on le grain sémantique d'après lequel on évalue l'identité de sens ? Dans le cas de conditions parfaitement identiques, comment se justifie l'existence de procédés concurrents et la construction effective des formes attestées? Quel est précisément le rôle des facteurs morphophonologiques dans la discrimination des schémas de construction concurrents ? Nous n'entrons pas dans le détail de ces questions ici, mais il apparaît que dans le cas des études sur -age, la concurrence morphologique est établie entre des procédés qui sélectionnent une même catégorie grammaticale de base et qui produisent à gros grain le même type de dérivé sémantique — la granularité grossière laissant ouverte la possibilité de mettre en évidence des différences de sens fines entre les formes concurrentes.
} 
les suffixes -age, -ment, -ion et -erie, et par Missud (2019), selon qui -age se distingue de la conversion par sa prédilection pour les verbes d'accomplissement.

On examine, d'autre part, l'existence de différences significatives entre les noms construits. Dubois (1962) note ainsi que -age, face à -ment, privilégie la dénotation de processus dans des domaines spécialisés, et s'impose diachroniquement dans la formation de noms d'action. Reprenant en partie les hypothèses défendues par Dubois (1962) et Kelling (2001), Martin (2010) prône une analyse multifactorielle, faisant intervenir la protoagentivité des N-age, la longueur de la chaîne événementielle décrite (impliquant plus de sous-événements pour -age que pour -ment), l'incrémentalité entre l'action décrite et le thème (toute partie de l'objet auquel s'applique le procès pouvant, dans le cas de -age vs -ment et -ion, être le thème d'un sous-événement du type décrit par le nom), et l'ontologie processive (-age décrivant plus volontiers que -ment et -ion des procès physiques). Cette dernière spécificité est confirmée par Fradin (2016), qui défend l'idée que -age se distingue de -ment par sa prédilection de construction avec des thèmes concrets, et par Wauquier et al. (2018), qui relèvent la tendance d'emploi de -age dans les domaines techniques et dans la dénotation d'actions concrètes, plus spécialisées que dans le cas de -ion. Enfin, selon Ferret et al. (2010), -age se distingue de -ée par l'encodage de l'aspect grammatical, les $\mathrm{N}$-age dénotant des procès dans leur déroulement (aspect imperfectif), tandis que les noms en -ée les présentent dans leur globalité (aspect perfectif).

Dans l'étude de la concurrence entre les N-age et les autres déverbaux, l'existence de doublets (ex. ajustage / ajustement, finissage / finition, arrivage / arrivée) attire l'attention des auteurs, et l'analyse des différences entre les types de dérivés se fonde souvent sur l'examen de ces paires de noms. Leur existence relève de différents cas de figure. Il se peut que les dérivés, qu'ils diffèrent ou non par leur type sémantique, soient construits sur des bases formellement identiques, mais présentant des différences linguistiques, en particulier syntaxiques ou sémantiques. Il arrive ainsi qu'en cas de polysémie verbale, la dérivation nominale opère sélectivement, comme dans le cas de montée vs montage, le premier étant dérivé d'un verbe de mouvement dont l'objet dénote un parcours, et le second d'un verbe de création dont l'objet dénote un produit. Un autre cas de figure est celui dans lequel les doublets sont construits sur le même lexème stricto sensu, i.e. sur une seule et même acception du vocable de base. Deux situations sont alors théoriquement possibles, selon que les formes des doublets relèvent ou non tendanciellement de deux types différents. Les situations de distinction récurrente sont propices à la mise en évidence de prédilections constructionnelles, et les cas d'équivalence résiduels peuvent être traités soit comme le résultat d'une neutralisation (dont les conditions exactes doivent être explicitées), soit comme des cas négligeables dus aux aléas de la constitution du lexique. Dans une conception classique des relations entre forme et sens dans la langue, on exclut la possibilité d'une synonymie persistante en diachronie (Aronoff, 2016), et l'examen des doublets est généralement mené dans le but de mettre en évidence des différences entre procédés concurrents. Cependant, les situations d'identité récurrente et étendue peuvent faire douter de l'existence de différences systématiques, et conduire à s'interroger sur la capacité de deux constructions concurrentes à se distinguer. Or selon Dubois (1962) et Fradin (2016), il existe des cas de doublets 
indistinguables entre noms suffixés en -age et en -ment (ex. engluage / engluement, ravalage / ravalement, triplage / triplement). De même, dans leur étude comparée des noms en -age et en -ment présents sur le web et dans les dictionnaires, Dal et al. (2018) recensent à la fois des cas de non-distinction (ex. désensablage / désensablement, relogeage / relogement, épinglage / épinglement), et des cas de distinction, mais dénués de régularité (-age et -ment s'échangeant les éventuels traits discriminants). Ils en concluent qu'il n'y a pas de principe distinctif de construction de déverbaux en -age et en -ment, et que les deux suffixes pourraient être considérés comme deux exposants d'une même règle de dérivation. On manque à ce jour de quantification précise sur les cas de surabondance morphologique observés, mais il existe différentes options théoriques pour en rendre compte, allant de l'hypothèse d'une surabondance systémique à celle d'un phénomène transitoire avant réduction des formes, en passant par celle d'une neutralisation locale des différences structurelles entre affixes distincts.

Deux remarques peuvent être faites ici. La première concerne la validité des inférences établies à partir de l'examen des doublets lexicalisés. Si ceux-ci semblent constituer un bon terrain d'observation de la concurrence dérivationnelle, la généralisation des différences relevées devrait se fonder sur des analyses de régularités observées à grande échelle. On peut en effet se demander si la spécialisation ne relève pas d'une contrainte de répartition lexicale qui ne tient pas à l'opération morphologique en soi, mais plutôt à une série d'idiosyncrasies liées à la lexicalisation, i.e. à la fixation de dénominations dans le lexique. La seconde remarque concerne le fait que les cas de doublets sont souvent observés en synchronie. Or le fait que deux constructions puissent être synchroniquement équivalentes démontre la capacité du système morphologique à générer des situations de surabondance, mais il n'exclut pas la tendance de celles-ci à se résorber en diachronie. La conception classique de la répartition lexicale (Bréal, 1897) est en effet une théorie diachronique : à un moment donné dans une langue donnée, les cas de surabondance peuvent tout à fait s'observer, sans pour autant contredire l'idée fondamentale qu'ils sont destinés à disparaître avec le temps.

\subsection{Multiplicité d'interprétations et polysémie}

La concurrence entre -age et les autres procédés de construction des noms déverbaux est principalement étudiée dans le cas des nominalisations d'action. Celles-ci n'épuisent cependant pas l'ensemble des significations que l'on peut associer à -age. Namer (2009) signale ainsi que -age peut régulièrement construire des noms d'action mais aussi de résultat, et Fradin (2012) indique pour -age les interprétations suivantes :

(1) a. Événement : atterrissage

b. Produit : pliage

c. Moyen : chauffage

d. Propriété : assemblage

e. Trajet : passage

f. Manière : tissage

g. Lieu : garage 


\section{h. Collectif : entourage}

i. Période : hivernage

En l'absence de définition et de tests identificatoires des catégories distinguées, on peut discuter l'étiquetage de certains cas. Il reste que la diversité sémantique des $\mathrm{N}$-age apparaît clairement, et qu'on peut relever la polysémie d'un certain nombre de noms, entre le sens visé dans (1) et celui d'action (ex. pliage, chauffage, assemblage, passage). L'existence de tels cas de polysémie conduit à s'interroger sur les mécanismes, morphosémantiques ou purement sémantiques, qui produisent les acceptions non actionnelles des $\mathrm{N}$-age.

Cette question est posée explicitement par Ferret et Villoing (2015), qui s’intéressent en particulier à la polysémie des $\mathrm{N}$-age dotés de la double interprétation d'action et d'instrument (ex. cirage, embrayage, barrage, camouflage). Ferret et Villoing soutiennent que la polysémie observée est le fruit d'une règle de construction morphologique, et non d'une figure sémantique (en l'occurrence métonymique). Les principaux arguments avancés sont les suivants :

(i) les procédés prototypiques de dérivation de noms d'instrument, tels que la suffixation en -eur et en -oir, ne génèrent pas d'ambiguité avec le sens d'action,

(ii) l'hypothèse d'une métonymie 'action' $\rightarrow$ 'instrument' ne permet pas d'expliquer la faible quantité observée de N-age instrumentaux, contrairement à l'hypothèse d'une contrainte sur la sélection verbale dans la règle de formation des noms d'instrument,

(iii) le fait que les noms d'action morphologiquement simples n'aient pas d'interprétation instrumentale contredit l'idée qu'il existe un schéma métonymique 'action' $\rightarrow$ 'instrument'.

Chacun de ces points est discutable. L'idée (i) selon laquelle il existe une règle indépendante de construction de noms d'instrument n'implique pas que cette règle s'applique au cas de -age, ni qu'il n'existe pas de construction métonymique 'action' $\rightarrow$ 'instrument'. L'argument (ii) est fragilisé par le fait que la métonymie dans le lexique peut être plus ou moins régulière (Apresjan, 1974 ; Copestake et Briscoe, 1995 ; Pustejovsky, 1995 ; Barque, 2008), et certes confiner parfois à la systématicité (comme dans le schéma 'contenant' $\rightarrow$ 'contenu', ex. verre, bol, assiette, cuillère, gamelle, etc.), mais aussi dans d'autres cas s'appliquer à très peu d'items lexicaux (comme dans le schéma 'meuble' $\rightarrow$ 'lieu', ex. bureau, bibliothèque). Par ailleurs, la contrainte d'instrumentalité pressentie pour le verbe de base peut être reproduite pour l'acception actionnelle du N-age dans l'hypothèse métonymique ${ }^{3}$.

\footnotetext{
${ }^{3}$ Ferret et Villoing expliquent la construction morphologique de $\mathrm{N}$-age instrumentaux (et sa faible productivité) par deux conditions : les verbes de base doivent vérifier les traits [+causation externe] et [+manière] - ce dernier correspondant à l'existence d'un actant instrumental dans la structure sémantique du verbe. Or ces deux conditions n'apparaissent ni comme suffisantes, ni comme nécessaires. D’une part, certains verbes dotés des traits [+causation externe] et [+manière] dérivent des noms actionnels mais non instrumentaux (ex. cisailler / cisaillage). D'autre part, la polysémie 'action' / 'instrument' existe pour certains noms dérivés de verbes qui n’ont pas de trait [+manière] (ex. invitation, convocation, attestation, autorisation, dont les verbes de base sont performatifs et n'impliquent pas la présence d'un instrument dans la réalisation des procès qu'ils décrivent).
} 
Enfin, (iii) l'inexistence de noms simples d'action également dotés du sens d'instrument ne suffit pas à prouver l'origine morphologique de la polysémie. L'absence de $\mathrm{N}$-age instrumental non polysémique pourrait à l'inverse affaiblir l'hypothèse défendue, étant donné que des procédés de construction polysémiques comme la dérivation de noms d'agent ou d'instrument en -eur permettent la formation de noms monosémiques de l'un ou l'autre type. L'origine de la polysémie des $\mathrm{N}$-age reste donc sujette à débat. Elle peut être questionnée à travers l'examen des $\mathrm{N}$-age néologiques, si l'on juge que ceux-ci témoignent des principes de construction productifs dans la grammaire des locuteurs.

\section{Méthodologie}

On peut se demander si les propriétés mises en évidence dans l'étude de la concurrence entre -age et les autres procédés de nominalisation, ainsi que dans l'étude de la polysémie des $\mathrm{N}$-age, s'observent dans les constructions néologiques en -age. Pour répondre à cette question, nous nous proposons d'analyser les propriétés syntaxiques et sémantiques d'un échantillon de $\mathrm{N}$-age néologiques, i.e. non recensés dans les ressources lexicales. L'étude proposée présente la limitation de ne pas comparer des noms déverbaux de différents types morphologiques. En revanche, elle ne se réduit pas à l'examen des $\mathrm{N}$-age pour lesquels il existe un doublet, et développe la réflexion sur l'héritage des propriétés syntaxiques et sémantiques entre bases verbales et dérivés nominaux (cf. Haas et al., 2008 ; Namer et Villoing, 2008 ; Ferret et al., 2010 ; Balvet et al., 2011 ; inter alia). Nous exposons dans cette section la méthode employée pour constituer l'échantillon d'étude, et les critères retenus pour analyser les verbes de base et les noms dérivés.

\subsection{Extraction des données}

Notre échantillon de $\mathrm{N}$-age néologiques est construit à partir du corpus $\mathrm{frWaC}$, qui est constitué de données du web recueillies dans le domaine «.fr» et qui contient 1,3 milliard de mots. Ce corpus est sélectionné en raison de sa taille importante, de sa date de constitution relativement récente ${ }^{4}$, et de la possibilité de consultation en libre accès. ${ }^{5}$

La constitution de l'échantillon étudié peut être décrite en quatre étapes ${ }^{6}$. Nous commençons par extraire de $\mathrm{frWaC}$ les formes se terminant par-age, filtrées par une série de critères additionnels tels que l'absence de caractères spéciaux ou de chiffres. Une première liste de 12421 items est ainsi obtenue. La deuxième étape consiste à

\footnotetext{
${ }^{4} \mathrm{Le}$ corpus $\mathrm{frWaC}$ a été constitué en 2010. D'autres corpus plus vastes et plus récents sont aujourd'hui disponibles, mais on peut penser que la plupart des $\mathrm{N}$-age néologiques extraits de $\mathrm{fr} W a \mathrm{C}$ ne se sont pas lexicalisés. Le point crucial ici est que nous sollicitons le corpus comme source pour l'extraction de néologismes candidats, et que ceux-ci font ensuite l'objet d'un contrôle dans la lexicographie actuelle (cf. infra).

${ }^{5}$ https://www.clarin.si/noske/run.cgi/first_form?_corpname=frwac;align

${ }^{6} \mathrm{Le}$ script d'extraction des données, la liste des noms échantillonnés et de leurs bases verbales, ainsi que le détail des annotations linguistiques et des analyses statistiques sont consultables en ligne à l'adresse suivante : https://github.com/neologisms-annotation/neologisms-ending-in-age.
} 
écarter les formes déjà lexicalisées. Nous éliminons les mots se terminant par -age présents dans les ressources Lefff (Sagot, 2010) et Lexique (New et al., 2001). La liste résultante est réduite à 9981 items potentiellement néologiques, mais elle est encore fortement bruitée. La troisième étape repose sur l'échantillonnage des items restants, ceux-ci étant trop nombreux pour être triés manuellement. Ils sont organisés par ordre de fréquence et séparés en 20 sous-listes d'environ 500 items chacune. Cent éléments sont tirés aléatoirement dans chaque sous-liste, de manière à obtenir un échantillon de 2000 formes. Cette méthode présente le double avantage de respecter la distribution des fréquences de la liste originelle, qui comprend une grande majorité d'items de fréquence 1 ou 2 , et de préserver l'éventail de fréquences initial - les néologismes ne se réduisant pas nécessairement à des hapax (dis)legomena.

Dans la quatrième et dernière étape, les 2000 items retenus sont triés manuellement afin d'éliminer ceux qui ne constituent pas des néologismes déverbaux en -age. Nous excluons d'abord les formes contenant une coquille ou une erreur typographique (1009 items). Sont ensuite écartés les noms comme flânage, rattachage, appointage, ceinturage, absents du Lefff et de Lexique, mais présents dans au moins un des deux dictionnaires de référence consultés - le Petit Robert (PR) et le Trésor de la langue française informatisé (TLFI). Les noms qui ne comprennent pas de suffixe -age sont également ignorés, qu'il s'agisse de noms non construits (putrilage), d'emprunts (advantage), de composés en -phage (lotophage), etc. Les noms suffixés en -age mais pour lesquels il n'existe pas de verbe attesté, ni dans les dictionnaires de référence, ni dans le corpus $\mathrm{r} W \mathrm{WaC}$, ne sont pas pris en compte, afin de garantir le caractère déverbal des noms conservés. Sont ainsi écartés les noms dont la dernière étape de construction est vraisemblablement une préfixation (surflicage), les noms dénominaux (confirmationage) et les noms désajectivaux (overbookage, considéré comme dérivé d'overbooké, en l'absence de verbe overbooker dans le corpus et les dictionnaires consultés).

Nous choisissons par ailleurs de ne pas prendre en compte les noms dont l'ensemble des occurrences relèvent de domaines spécialisés, comme moisage (charpenterie), débillardage (industrie), carassonage (viticulture), désatrocage (ostréiculture), gansage (textile), planchage (philatélie), dans l'idée que ceux-ci ne sont pas nécessairement néologiques. Le caractère spécialisé d'un $\mathrm{N}$-age est déterminé soit d'après l'étiquetage de sa base verbale dans les dictionnaires de référence comme terme de spécialité, soit d'après l'examen des occurrences du nom en contexte. Au total, 251 déverbaux en -age de spécialité sont ainsi écartés, ce qui semble confirmer la tendance précédemment relevée des $\mathrm{N}$-age à l'emploi terminologique.

Ce tri manuel aboutit à une liste de 139 déverbaux néologiques en -age qui seront ensuite annotés (et se déclineront alors en 182 acceptions). Une limite de la méthode employée est de ne pas dépister les néosémies construites à partir de noms lexicalisés, ni les néologismes morphologiques qui auraient la même forme qu'un lexème existant. Les rares exceptions ici sont les noms qui ne sont filtrés qu'à la dernière étape du processus et pour lesquels il apparaît que le sens enregistré dans les dictionnaires n'est pas celui observé en corpus, comme dans le cas de lattage au sens de 'action de frapper quelqu'un' ( $v s$ 'action de garnir de lattes' 
et 'ouvrage de lattes') et d'évitage au sens de 'action d'éviter' ( $v s$ '[marine] changement de direction du cap d'un navire sous l'effet du vent ou de la marée').

Le nombre final de 139 formes parait faible, comparativement au nombre de candidats initial. L'étude que nous présentons doit à cet égard être considérée comme une première exploration, à confirmer le cas échéant sur des données plus abondantes. Il n'en reste pas moins que le caractère aléatoire de notre échantillonnage garantit une certaine représentativité, et que le nombre de 139 formes / 182 acceptions permet d'effectuer des analyses statistiquement significatives pour la plupart des propriétés étudiées (cf. infra).

\subsection{Annotation des verbes de base}

Nous partons de l'idée que les bases des opérations morphologiques sont des unités spécifiées sémantiquement (Mel'čuk, 1993 ; Fradin et Kerleroux, 2003), et nous apparions des lexèmes verbaux et nominaux, ceux-ci étant considérés comme des acceptions dotées de leur propre structure syntaxique et sémantique. En cas de polysémie, l'appariement se fait au plus proche, en vertu d'un postulat de correspondance sémantique entre base et dérivé, c'est-à-dire que les lexèmes analysés comme bases de dérivation sont ceux dont le sens se rapproche le plus de celui des dérivés. Ainsi, semage 'action d'ensemencer' est mis en relation morphologiquement avec semer ${ }_{1}$ 'mettre en terre des semences', et non avec semer $_{2}$ 'se débarrasser de la compagnie de quelqu'un'. L'appariement peut donner lieu à différentes configurations en cas de polysémie nominale, selon que les acceptions de noms distinguées sont ou non liées au même lexème verbal. Par exemple, les deux acceptions de fusionnage dans (2), comme 'action de fusionner' et 'résultat de l'action de fusionner', sont liées à un même lexème fusionner, tandis que les deux acceptions de chahutage dans (3) sont liées à deux chahuter distincts, l'un intransitif, au sens de 'faire du chahut', et l'autre transitif, au sens de 'malmener'.

(2) a. Et puis même s'il y avait eu un sujet existant, on aurait simplement procédé à un fusionnage des sujets, c'est tout. (web)

b. Y aurait-il une solution pour supprimer ce fusionnage ? (web)

(3) a. Miss Lignac restait pour surveiller les enfants et les calmer s'il y avait du chahutage. (web)

b. Jean-Michel Baylet avait déjà déploré le chahutage du philosophe Alain Finkielkraut lors de sa visite à Nuit Debout. (web)

Notons que différents lexèmes verbaux sont également distingués en cas d'existence d'une forme réfléchie lexicalisée du verbe, i.e. une forme syntaxiquement ou sémantiquement autonome (cf. Boons et al., 1976 ; Creissels, 2007 ; Barque et al., 2019). L'appariement morphologique est établi en conséquence. Par exemple, les deux acceptions d'applicage dans (4) sont appariées respectivement à appliquer et à s'appliquer :

(4) a. Au moment de l'applicage, la texture n'est pas huileuse (c'est parfait). (web)

b. Je dirais que c'est aussi drôle que c'est mal dessiné. Ça aurait mérité un peu d'applicage. (web) 
Les informations renseignées pour chaque base verbale sont les suivantes :

- l'attestation du lexème visé dans le PR ou le TLFI,

- la présence du lexème visé dans le corpus de référence $(f r W a C)$,

- la transitivité,

- le nombre d'arguments,

- les rôles sémantiques assignés aux différents arguments,

- la classe aspectuelle.

Précisons que nous annotons strictement la transitivité directe des verbes. Les rôles sémantiques sont encodés d'après une classification élémentaire, comprenant les rôles d'agent, patient, thème, bénéficiaire, instrument, expérienceur, stimulus, siège et locatif. L'aspect lexical est décrit selon les quatre classes vendleriennes, auxquelles nous ajoutons celle des achèvements graduels (" degree achievements »), qui correspond aux verbes exprimant des changements d'état gradables, comme rétrécir ou désencrasser, et dont la télicité est intrinsèquement variable (Dowty, 1979 ; Bertinetto et Squartini, 1995 ; Hay et al., 1999 ; Huyghe 2015a). Les différentes classes verbales sont identifiées d'après les conditions suivantes : les verbes d'état ne se construisent pas avec être en train de, contrairement aux quatre autres classes qui, elles, se distinguent par leur prédilection d'emploi avec les compléments de durée introduits par pendant vs en (verbes d'activité), en vs pendant (verbes d'accomplissement), par l'emploi également favorisé avec les deux types de compléments (verbes d'achèvement graduel), ou par l'incompatibilité avec s'arrêter de et avec les compléments de temps en en ne décrivant pas une phase préparatoire de l'action (verbes d'achèvement). L'encodage de l'aspect lexical que nous proposons est établi par défaut, i.e. indépendamment des éventuelles coercitions contextuelles dues à la présence d'un objet non délimité (Verkuyl, 1993 ; Rothstein, 2004). Les verbes transitifs sont ainsi testés en présence d'objets délimités : par exemple, résumer est catégorisé comme un accomplissement, en vertu du fait que résumer un livre est télique (vs résumer des livres, qui est coercé et atélique).

\subsection{Annotation des noms dérivés}

Les $\mathrm{N}$-age sont annotés à partir de leurs occurrences dans le corpus $\mathrm{fr} W a \mathrm{C}$, étendues à celles que l'on peut rencontrer actuellement sur l'ensemble du web. Cette extension permet de préciser la polysémie de certains noms, et d'affiner l'annotation en mettant à l'épreuve certaines constructions mobilisées dans les tests d'analyse des données.

Les informations renseignées pour chaque $\mathrm{N}$-age sont les suivantes :

- le type ontologique,

- le type relationnel,

- le nombre d'arguments,

- les rôles sémantiques assignés aux différents arguments,

- la classe aspectuelle,

- l'existence ou non d'une autre acception dans l'échantillon analysé. 
À la suite de Huyghe (2015b), nous distinguons entre deux formes de typologie sémantique des noms : une typologie ontologique, établie d'après la partition du réel de référence, et une typologie relationnelle, reposant sur la description d'un rôle prédicatif ou d'une fonction référentielle. La typologie sémantique employée comprend 10 types ontologiques (animé, objet, objet cognitif, action, domaine, institution, propriété, temps, mesure, phénomène), dont 4 ont été de facto utilisés dans l'annotation des N-age (action, propriété, objet et objet cognitif). Ces types sont identifiés à l'aide de tests proposés dans la littérature (Godard et Jayez, 1996 ; Flaux et Van de Velde, 2000 ; Kleiber et al., 2012 ; Huyghe, 2015b ; inter alia). Les noms d'action peuvent être sujet de avoir lieu, se produire ou objet de effectuer, accomplir, procéder à ; les noms de propriété peuvent s'employer dans les expressions être d'un grand $N$, état de $N$, ou comme objet de ressentir, éprouver, faire preuve de ; les noms d'objet peuvent être sujet de se trouver suivi d'un complément de localisation physique, mais pas de décider de, choisir de, prendre soin de ; les noms d'objet cognitif peuvent s'employer dans des expressions telles que le $N$ selon lequel $P$, un $N$ décousu, un $N$ difficile à admettre.

Les types relationnels, pour leur part, correspondent aux rôles sémantiques argumentaux considérés dans l'annotation des verbes (cf. supra), auxquels s'ajoute un type résultatif. L'avantage de distinguer types ontologiques et types relationnels est d'éviter la confusion entre les niveaux sémantiques analysés, et de permettre de repérer différentes combinaisons de description intrinsèque et relationnelle. Un résultat peut ainsi être un objet (5a), un objet cognitif (5b) ou une propriété $(5 \mathrm{c})$ :

(5) a. Au Bosquet, les chics de la société boivent des concoctages originaux. (web) b. Beaucoup d'histoires sont des blablatages. (web)

c. Nous sommes rassurés : l'avion est toujours là ! Le « rassurage » est de courte durée. (web)

S'agissant de la complémentation, une des particularités des noms est de ne pas nécessairement (et dans les faits, très rarement) se construire conjointement avec l'ensemble de leurs arguments. L'emploi de constructions nominales comprenant un seul argument, typiquement patient (6a) ou agent (6b), est nettement plus fréquent en corpus que celui de SN biargumentaux (6c) $:^{7}$

(6) a. (...) le bastonnage des mères venues réclamer leur enfant (web)

b. (...) les dérapages anciens, Métro Charonne, le bastonnage des motards voltigeurs (web)

c. (...) au-delà du simple bastonnage de monstres par des héros et héroïnes (web)

En conséquence, nous avons choisi d'annoter les structures argumentales des noms d'après leur extension maximale, en tenant compte de l'ensemble des rôles

\footnotetext{
${ }^{7} \grave{A}$ titre indicatif, une recherche des séquences «bastonnage de ", « bastonnage des » et « bastonnage du » sur Google.com le 08/07/20 permet de dénombrer, pour un total de 190 attestations pertinentes, 13 constructions biargumentales et 177 constructions monoargumentales.
} 
qu'ils peuvent assigner, indépendamment de la cooccurrence effective de ces arguments en corpus.

Les catégories d'aspect lexical, quant à elles, s'appliquent uniquement aux noms d'éventualité, i.e. aux noms typés ontologiquement comme noms d'action ou de propriété. L'aspect nominal a été encodé selon la classification retenue pour le domaine verbal, à l'aide de tests présentés dans Haas et al. (2008). Sont ainsi catégorisés comme noms d'état les noms d'éventualité qui ne peuvent se construire avec aucun verbe support dynamique (avoir lieu, effectuer, accomplir, procéder à, commettre, etc.). Les noms d'activité sont les noms d'action compatibles avec les expressions de durée (un $N$ de $x$ temps, $x$ temps de $N$ ), qui sélectionnent préférentiellement des compléments de durée introduits par pendant vs en, et qui ne satisfont pas le test du paradoxe imperfectif adapté aux noms (ex. Leur baladage dans la forêt a été interrompu implique Ils se sont baladés). Les noms d'accomplissement se distinguent des noms d'activité par le fait qu'ils sélectionnent préférentiellement des compléments de durée introduits par en vs pendant, et satisfont le test du paradoxe imperfectif adapté aux noms (ex. Le transmettage de l'information a été interrompu n'implique pas L'information a été transmise). Les noms d'achèvement graduel sont des noms d'action compatibles avec les expressions de durée, sélectionnant aussi bien les compléments de durée introduits par en que ceux introduits par pendant, et pouvant s'employer dans un fort $N$, un degré de $N$, un certain $N$ avec une interprétation intensive. Enfin, les noms d'achèvement sont des noms d'action qui s'emploient difficilement avec l'ensemble des expressions de durée.

Comme dans le cas des verbes, l'aspect est annoté par défaut, en testant les noms qui nécessitent un complément accompagnés d'un argument interne délimité, dans le but d'éviter les effets de coercition interprétative causés par la présence d'arguments non délimités (ex. le transmettage d'une information vs le transmettage d'informations).

Une situation de polysémie est postulée dès lors qu'une des propriétés annotées pour un N-age donné varie. Ainsi, confinage se voit associer deux acceptions, selon qu'il décrit une action (7a) ou un objet locatif (7b), et retraçage se voit assigner deux acceptions, selon qu'il prend pour argument un patient (8a) ou un thème (8b) :

(7) a. Le président américain s'est exprimé sur Twitter, se félicitant de la «bonne construction » du bâtiment qui a facilité le confinage de l'incendie. (web)

b. Quelqu'un aurait en photos comment bricoler un confinage pour les greffes? (web)

(8) a. La réhabilitation comprend aussi le retraçage des allées. (web)

b. Le fait que ces machines soient également nombreuses et disséminées dans plusieurs pays du monde rend difficile le retraçage de l'origine de l'attaque. (web)

\section{Analyse des données}

Cette section présente une série d'observations et d'analyses effectuées à partir de l'annotation des 139 néologismes en -age que nous avons échantillonnés (et qui 
Tableau 1. Rôles sémantiques assignés par les bases verbales des $\mathrm{N}$-age néologiques

\begin{tabular}{ccccccc}
\hline Agent & Patient & Thème & Locatif & Expérienceur & Bénéficiaire & Siège \\
\hline 145 & 72 & 54 & 17 & 5 & 4 & 1 \\
\hline
\end{tabular}

Tableau 2. Aspect lexical des bases verbales des $\mathrm{N}$-age néologiques

\begin{tabular}{ccccc}
\hline Activité & Achèvement & Accomplissement & Ach. graduel & État \\
\hline 65 & 46 & 31 & 10 & 4 \\
\hline
\end{tabular}

correspondent à 182 acceptions nominales). Nous décrivons les propriétés des verbes de base et des noms dérivés, avant de nous intéresser à la polysémie des $\mathrm{N}$-age et à leurs conditions d'émergence dans le discours. L'absence de comparaison entre suffixes formateurs de noms déverbaux ne nous permet pas d'évaluer les hypothèses relevant de la concurrence entre -age, -ment, -ion, -erie, etc. Un certain nombre de points soulevés dans la littérature peuvent néanmoins être discutés. Nos analyses confirment certaines propriétés de construction fondamentales précédemment relevées, mais font aussi apparaître la spécificité des néologismes en -age quant à la sélection et la préservation des propriétés de leurs bases.

\subsection{Bases verbales}

Nos données comprennent 156 lexèmes verbaux de base pour l'ensemble des acceptions nominales annotées, dont 117 sont transitifs et 39 intransitifs. En comparaison, la ressource Les verbes français (Dubois et Dubois-Charlier, 1997) recense 19580 entrées de verbes ayant au moins un emploi transitif contre 6029 intransitifs (au sens où nous l'entendons ici). Le test du khi carré de Pearson montre que la différence entre les deux distributions n'est pas significative $\left(\chi^{2}(1, N=25765)=0,1829, p=0,668856\right)$, autrement dit que -age ne sélectionne pas significativement plus de verbes transitifs que de verbes intransitifs par rapport à la distribution de ces deux catégories dans l'ensemble du lexique (tout au moins tel qu'analysé dans Les verbes français). Ce résultat ne compare certes pas la sélection des bases entre les différents procédés de nominalisation, mais il ne montre pas a priori de prédilection de -age pour les bases transitives, contrairement à ce qui est parfois inféré des études qui comparent les différents procédés de construction de noms déverbaux.

La distribution des rôles sémantiques assignés par les verbes annotés est présentée dans le tableau 1.

Le rôle qui domine est celui d'agent (attribué au sujet), suivi de ceux de patient et de thème, les sièges et expérienceurs étant très largement minoritaires. Il apparaît ainsi que -age sélectionne principalement des verbes dynamiques, conformément à ce qui est indiqué dans l'ensemble des travaux sur le sujet. Cette propriété est confirmée par l'examen des classes aspectuelles des verbes de base (tableau 2).

La catégorie la plus représentée est celle des activités, bien que les procès téliques (accomplissements et achèvements regroupés) soient majoritaires. Ces résultats 
Tableau 3. Correspondance entre types ontologiques et relationnels des $\mathrm{N}$-age néologiques

\begin{tabular}{lccccc}
\hline & Action & Propriété & Objet & Obj. cognitif & Total \\
\hline$\varnothing$ & 152 & 4 & - & - & 156 \\
\hline Résultat & - & 8 & 7 & 8 & 23 \\
\hline Instrument & - & - & 1 & 1 & 2 \\
\hline Locatif & - & - & 1 & - & 1 \\
\hline Total & 152 & 12 & 9 & 9 & 182 \\
\hline
\end{tabular}

contrastent avec ceux observés par Missud (2019) pour les N-age non néologiques (parmi les $100 \mathrm{~N}$-age analysés, 52 dérivent de verbes d'accomplissement, 23 de verbes d'achèvement et 22 seulement de verbes d'activité), ce qui laisse entrevoir une variation diachronique ou un effet de lexicalisation important dans la construction des $\mathrm{N}$-age - les noms analysés par Missud étant des formes de haute fréquence en français.

\subsection{Noms dérivés}

L'échantillon analysé contient 182 acceptions de N-age néologiques, pour 139 formes différentes (soit un taux de polysémie de 1,31). L'analyse des types ontologiques décrits par ces noms indique une nette prédilection pour la description d'actions : 152 noms analysés sont des noms d'action, 12 des noms de propriété, 9 des noms d'objet et 9 des noms d'objet cognitif. Cette prédilection confirme les observations de Wauquier et al. (2018) concernant la proximité de comportement distributionnel des $\mathrm{N}$-age et des noms d'action en général.

Dans 156 cas, le type relationnel est non marqué. L'éventualité décrite par le nom correspond à celle décrite par le verbe, et le nom hérite donc du type ontologique verbal. Parmi les cas marqués, on dénombre 23 noms de résultat, 2 noms d'instrument et 1 nom de locatif. La correspondance entre les types ontologiques et relationnels est présentée dans le tableau 3.

Dans le cas des noms d'éventualité non marqués relationnellement, les structures argumentales et les grilles thématiques sont intégralement héritées. Les rôles assignés par les $\mathrm{N}$-age sont donc majoritairement ceux d'agent, de patient et de thème, même si comme nous l'avons relevé précédemment, la pluralité argumentale se rencontre rarement en discours. Nous n'avons pas quantifié les différents types d'occurrences, mais l'examen approfondi des données de corpus nous semble indiquer une prédilection assez nette pour la construction avec l'argument interne (patient ou thème) plutôt qu'externe (agent).

La classification aspectuelle des noms d'éventualité non marqués relationnellement montre elle aussi un héritage intégral des propriétés verbales. Si l'on prend également en considération les noms d'éventualité marqués - qui sont par ailleurs tous possiblement construits par métonymie, en ce sens qu'il existe une acception actionnelle attestée pour chacun d'entre eux - on dénombre 8 cas de décalage aspectuel entre bases et dérivés, les premiers étant des verbes dynamiques, et les 
seconds des noms de propriété (résultante). La proportion de cas de décalage reste significativement inférieure à celle observée dans la ressource Nomage (Balvet et al., 2011), dans laquelle 12 cas de divergence aspectuelle sont dénombrés pour un total de $56 \mathrm{~N}$-age d'éventualité (non néologiques) annotés $\left(\chi^{2}(1, \mathrm{~N}=220)=\right.$ $13,8362, p=0,000199)$. On peut donc formuler l'hypothèse que les décalages d'aspect observés dans le cas des nominalisations en -age ne sont pas le fait de la construction morphologique, mais plutôt du processus de lexicalisation, celui-ci incluant toutes sortes de contingences liées à la répartition des significations dans le lexique, aux nécessités dénominatives, aux blocages lexicaux, aux variations diachroniques, etc.

Notons ici que la consultation des données en corpus indique une tendance assez nette des $\mathrm{N}$-age à apparaître dans des emplois non occurrentiels, dans lesquels les noms dénotent génériquement un type d'action plutôt qu'un événement spécifique. Par exemple, des noms comme pistonnage, googlage et spoilage se rencontrent plus fréquemment dans des exemples comme (9a), (10a), (11a) que dans des exemples comme (9b), (10b), (11b) :

(9) a. Je suis pour qu'elle fasse des books et qu'elle aille dans des associations, car j'aime pas le pistonnage. (web)

b. Des relations de famille peuvent bien sûr expliquer des pistonnages. (web)

(10) a. Décidément, le googlage est devenu quasi-systématique. (web)

b. Un googlage de mon nom risquerait de me disqualifier. (web)

(11) a. Le spoilage est interdit en cette période de surtension! (web)

b. Dans ce cas-là, on peut s'attendre à un spoilage dès $15 \mathrm{~h} 30$. (web)

Le mode d'annotation que nous avons adopté ne permet pas de quantifier cette prédilection d'usage. Il faudrait étendre l'analyse et comparer les tendances d'emploi de l'ensemble des déverbaux d'éventualité pour pouvoir établir une éventuelle corrélation entre la forme des nominalisations et leur capacité d'interprétation générique, comme type d'action ou d'activité.

\subsection{Polysémie}

L'échantillon de $\mathrm{N}$-age néologiques analysés contient 40 formes ambiguës (soit $28,8 \%$ des formes analysées) qui se répartissent en 83 acceptions. Les combinaisons de types ontologiques et relationnels observées sont présentées dans les tableaux 4 et $5^{8}$.

Les associations de types ontologiques mettent quasiment toujours en jeu une acception actionnelle, la seule exception étant la combinaison des sens d'objet et d'objet cognitif pour le nom mitonage, qui a en fait 4 acceptions et inclut également des combinaisons d'action et d'objet (cognitif), dans ses interprétations comme 'préparation culinaire' ou 'mensonge'. Les associations de types relationnels, pour leur part, font apparaître une nette prédilection pour les combinaisons de cas non marqués (typiquement entre deux types d'actions, ex. disjonctage dans le

\footnotetext{
${ }^{8}$ Les chiffres présentés dans chaque tableau correspondent au nombre de formes nominales dotées de deux acceptions dont le type est indiqué en ligne et en colonne.
} 
Tableau 4. Combinaisons de types ontologiques pour les $\mathrm{N}$-age polysémiques

\begin{tabular}{|c|c|c|c|c|}
\hline & Action & Propriété & Objet & Obj. cognitif \\
\hline Action & 16 & 9 & 11 & 10 \\
\hline Propriété & 9 & - & - & - \\
\hline Objet & 11 & - & - & 1 \\
\hline Obj. cognitif & 10 & - & 1 & - \\
\hline
\end{tabular}

Tableau 5. Combinaisons de types relationnels pour les $\mathrm{N}$-age polysémiques

\begin{tabular}{|c|c|c|c|c|}
\hline & $\varnothing$ & Résultat & Instrument & Locatif \\
\hline$\varnothing$ & 17 & 26 & 2 & 1 \\
\hline Résultat & 26 & 1 & - & - \\
\hline Instrument & 2 & - & - & - \\
\hline Locatif & 1 & - & - & - \\
\hline
\end{tabular}

Tableau 6. Combinaisons de types sémantiques pour les $\mathrm{N}$-age polysémiques liés à une même acception verbale

\begin{tabular}{ccccccc}
\hline & Prop-Résult & Cog-Résult & Obj-Résult & Cog-Instru & Obj-Instru & Obj-Loc \\
\hline Action- $\varnothing$ & 8 & 8 & 7 & 1 & 1 & 1 \\
\hline
\end{tabular}

sens de 'interruption de courant' et et de 'perte du contrôle de soi') et de cas non marqué et de résultat (typiquement entre une action et son résultat, ex. concoctage dans le sens de 'action de concocter' et de 'objet concocté').

Il existe différentes configurations d'appariement polysémique entre verbes et noms dérivés. Deux acceptions d'une même forme nominale peuvent être soit liées à la même acception verbale (ex. entassage ${ }_{1}$ 'fait d'entasser des objets' et entassage ${ }_{2}$ 'objets entassés', tous deux associés à entasser 'mettre en tas'), soit liées à des acceptions verbales distinctes (ex. empruntage ${ }_{1}$ 'fait d'obtenir à titre de prêt' et empruntage $_{2}$ 'fait de suivre une voie', associés respectivement à emprunter ${ }_{1}$ 'obtenir à titre de prêt' et emprunter ${ }_{2}$ 'suivre une voie'). Dans les cas où les acceptions nominales sont liées à une même acception verbale, on observe que le nom a toujours au moins une acception actionnelle non marquée relationnellement. La distribution des acceptions associées à ce sens d'action est présentée dans le tableau 6.

À quelques rares exceptions près, les cas de polysémie nominale liés à une même acception verbale sont du type 'action' / 'résultat'. Ils correspondent à un schéma de polysémie répandu parmi les nominalisations (cf. Grimshaw, 1990 ; Jacquey, 2006 ; Melloni, 2011 ; inter alia).

Plusieurs questions se posent quant à la polysémie entre noms d'action et noms de résultat. On peut se demander notamment (i) s'il existe des facteurs qui 


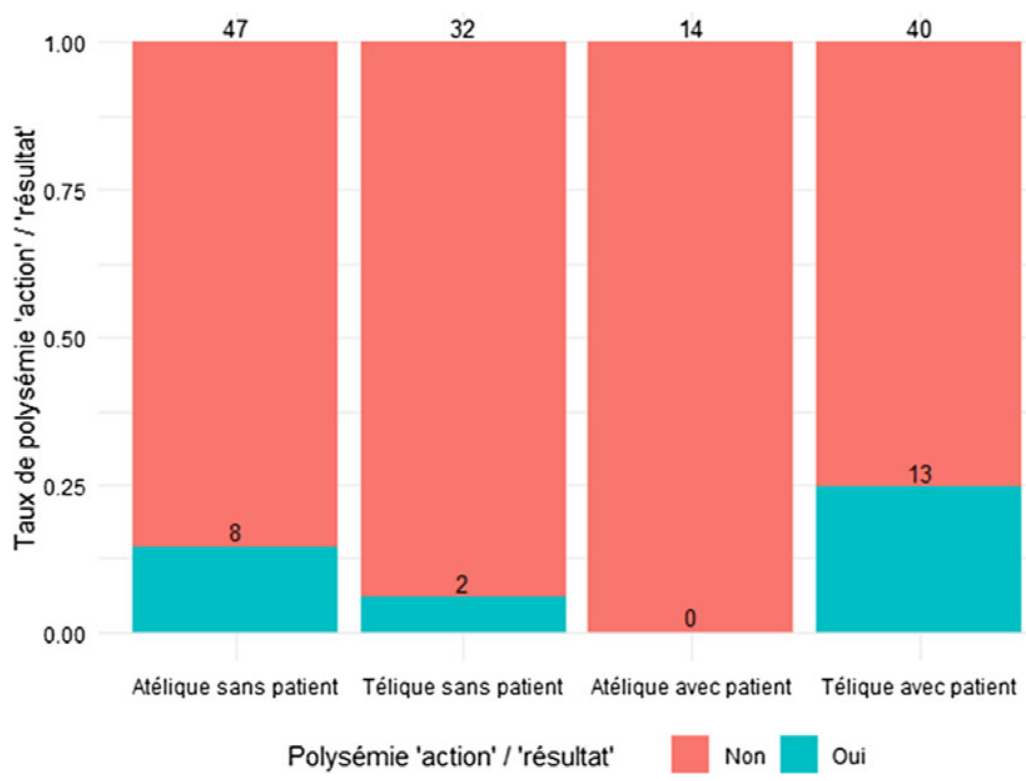

Figure 1. Proportions de verbes (non) téliques et (non) patientifs dérivant des N-age polysémiques 'action' / 'résultat'.

permettent de prédire l'existence pour un verbe donné d'une double nominalisation d'action et de résultat, et (ii) si la construction de l'acception résultative se fait par dérivation ou par métonymie, ce qui revient à se demander si elle a pour origine le verbe lui-même ou sa nominalisation actionnelle.

S'agissant de (i), deux prédicteurs peuvent être envisagés. Le premier est la présence d'un patient dans la structure argumentale du verbe, l'affectation de l'objet pouvant conduire à un résultat saillant. Le second est la télicité du verbe, la culmination de l'action pouvant favoriser la résultativité. Nous avons analysé ces deux facteurs dans une régression logistique, qui permet d'évaluer leur effet sur l'existence d'une polysémie nominale 'action' / 'résultat' pour un verbe donné. La significativité des prédicteurs est déterminée par un test de khi carré qui compare un modèle avec et un modèle sans le prédicteur. Il apparaît que les propriétés testées n'ont pas d'effet simple, car ni la présence d'un patient $\left(\chi^{2}(1, N=156)=2,0068\right.$, $\mathrm{p}=0,1566)$ ni la télicité $\left(\chi^{2}(1, \mathrm{~N}=156)=0,99387, \mathrm{p}=0,3188\right)$ n'ont d'effet significatif sur l'actualisation d'une polysémie 'action' / 'résultat'. Leur interaction, cependant, donne lieu à un effet significatif $\left(\chi^{2}(1, \mathrm{~N}=156)=8,3851, \mathrm{p}=0,003783\right)$. En effet, les quatre configurations issues de la combinaison des deux propriétés (i.e. avec patient et atélique, sans patient et atélique, avec patient et télique, sans patient et télique) sont associées à des quantités d'occurrences de la polysémie nominale 'action' / 'résultat' significativement différentes les unes des autres. Les données correspondantes observées dans notre échantillon sont présentées dans la figure 1.

Plus précisément, le modèle de régression logistique prédit qu'un verbe atélique sans patient a 14,6\% de chance de donner lieu à une polysémie 'action' / 'résultat', un 
verbe atélique avec patient $0,0 \%$, un verbe télique sans patient $5,9 \%$, et un verbe télique avec patient $24,5 \%$. Dans nos données, 23 verbes sur 156 conduisent à une polysémie 'action' / 'résultat', ce qui correspond à un taux de $14,7 \%$. Un verbe possédant les deux propriétés testées est donc plus favorable à la polysémie visée, ce qui confirme (en partie au moins) notre hypothèse de départ. ${ }^{9}$

S'agissant de (ii), les éléments dont nous disposons pour l'instant ne permettent pas de démontrer si la construction morphologique ou métonymique est à l'origine des acceptions résultatives. On peut cependant noter qu'en cas de néologie en -age, les acceptions résultatives sont toujours accompagnées d'acceptions actionnelles. Plus généralement, nous n'observons pas d'acception marquée relationnellement pour une forme nominale en -age (exprimant le résultat, l'instrument ou le locatif) qui n'ait pas également une acception non marquée, c'est-à-dire une acception décrivant la même action ou le même état que le verbe de base. Cet argument ne suffit certes pas à démontrer l'hypothèse métonymique, mais il satisfait une condition nécessaire de cette hypothèse, en ce sens qu'une construction métonymique du sens résultatif, instrumental ou locatif implique l'attestation du sens actionnel. Il peut donc être considéré comme un indice en sa faveur.

\subsection{Motivation de la création néologique}

En complément de l'analyse des propriétés sémantiques et syntaxiques présentée ci-dessus, on peut s'interroger sur les raisons de l'apparition en discours de déverbaux néologiques en -age. Nous faisons état de quelques observations à ce sujet, recueillies lors de l'annotation des données. Il s'agit ici de distinguer qualitativement les principaux cas de figure rencontrés, et d'apporter quelques éléments de réflexion sur les structures du lexique et sur l'organisation des familles morphologiques constituées autour de verbes.

Comme on peut s'y attendre, une des fonctions essentielles des $\mathrm{N}$-age néologiques est de combler une lacune lexicale, qu'il existe ou non d'autres noms déverbaux dans la famille du nom créé. Des noms comme véhiculage 'action de véhiculer quelqu'un ou quelque chose', cachage 'action de cacher quelque chose', paumage 'action de paumer quelque chose', molestage 'action de molester quelqu'un', éteignage 'action d'éteindre quelque chose' sont dérivés de verbes qui n'ont pas de nom d'éventualité lexicalisé dans leur famille morphologique. À l'inverse, un nom comme assistage a des concurrents morphologiques, mais

\footnotetext{
${ }^{9}$ Il reste qu'un verbe ne possédant aucune des deux propriétés semble plus favorable à la polysémie qu'un verbe n'en ayant qu'une seule, ce qui est a priori surprenant. Cependant, une analyse de la régression logistique par comparaison deux à deux des catégories de verbes, effectuée à partir du package lsmeans dans l'environnement $R$ (R Core Team, 2015), ne révèle pas de différence significative entre les verbes atéliques non patientifs et les verbes atéliques patientifs $(p=0,9881)$ ou téliques non patientifs $(p=0,2235)$. Le fait de n'avoir aucune des deux propriétés n'a donc pas forcément d'effet sur la capacité d'un verbe à produire des $\mathrm{N}$-age d'action / résultat. En ce qui concerne les cas recensés dans nos données, on peut noter que les verbes atéliques non patientifs qui dérivent des $\mathrm{N}$-age sont soit des verbes qui impliquent un état affectant le sujet (ex. psychoter, comater), soit des verbes de parole, corrélés à la description d'objets cognitifs qui ne sont toutefois pas dénotés comme arguments, et ne jouent donc pas le rôle de patient (ex. complimenter, blablater, mitonner, bullshiter).
} 
ceux-ci ne s'emploient pas dans le sens visé par le néologisme - assistance ne conviendrait pas dans (12).

(12) Pour moi ce soir, c'est « assistage » au spectacle chorégraphié de l'album de Philippe Katerine. (web)

On peut penser que dans certains cas, la création d'un $\mathrm{N}$-age permet de souligner un trait sémantique non saillant dans les noms concurrents. Il n'est pas exclu que réveillage dans (13) soit préféré à réveil, qui est polysémique et non nécessairement intentionnel, pour faire saillir l'agentivité de l'action, selon une propriété sémantique prototypiquement associée aux $\mathrm{N}$-age :

(13) 9h09 : tentative de réveillage des autres, échec cuisant. (web)

La formation d'un N-age peut également être motivée par la nominalisation d'une expression verbale phraséologique, que celle-ci soit figée (14a), ou simplement collocationnelle (14b) :

(14) a. Chez moi, c'est Monsieur qui est champion du monde de faisage de tronche. (web)

b. C’est mon arrêtage de fumer qui me rend acerbe, aigri et frustré ! (web)

En dehors des cas où la création d'un N-age se justifie par l'absence d'expression adéquate pour exprimer la pensée du locuteur, on trouve de nombreuses occurrences de $\mathrm{N}$-age néologiques difficilement distinguables de formes établies dans le lexique. Ainsi tutoyage, supprimage et musculage dans (15) peuvent-ils commuter avec tutoiement, suppression et musculation:

(15) a. Le Daily City a le plaisir de vous annoncer que dès à venir le tutoyage sera de rigueur entre les journalistes. (web)

b. Tout non-respect sera sanctionné d'un supprimage du topic. (web)

c. Il va falloir que je fasse un peu de musculage parce que c'est vraiment dur ! (web)

Il en va généralement de même pour arnaquage et arnaque, surpeuplage et surpeuplement, rêvassage et rêvasserie, insérage et insertion, citage et citation, chicanage et chicanerie, validage et validation, etc. Le risque de surabondance (Thornton, 2012) ne semble donc pas bloquer la formation néologique de déverbaux en -age.

Notons enfin que la création en discours d'un N-age peut avoir une visée ludique, en contribuant aux jeux de langue, notamment dans les effets de série ou de " rafale » relevés par Dal et Namer (2016) :

(16) a. Merci de ton passage, et de ton reviendage ! (web)

b. Après le soufflage des bougies et le dégustage du divin gâteau, un baladage s'imposait. (web)

c. Papotage, copinage, discutage, mangeage (Vérigoud, comme dit Chuck Berry) et reposage. (ex. cité dans Dal et Namer, 2016: 11) 
Dans ce cas de figure, la proximité de N-age lexicalisés facilite l'emploi d'un néologisme occasionnel, s'insérant dans une série dérivationnelle constituée ad hoc.

\section{CONCLUSION}

Dans cet article, nous avons étudié les propriétés sémantiques et syntaxiques d'un échantillon de $\mathrm{N}$-age néologiques et de leurs bases verbales. L'analyse détaillée des néologismes, par contraste avec celle des mots construits lexicalisés, permet d'approcher les propriétés des opérations morphologiques en soi (Corbin, 1987 ; Plag, 1999), et notamment de distinguer entre sémantique dérivationnelle (constituée des opérations sémantiques associées aux procédés morphologiques) et sémantique lexicale (correspondant à l'ensemble des propriétés sémantiques des unités en langue).

Les résultats de l'étude viennent confirmer certaines propriétés de la suffixation en -age déjà relevées dans la littérature, telles que la formation très majoritaire de noms d'action à partir de verbes dynamiques ou l'emploi favorisé dans les domaines de spécialité. Cependant, ils contredisent également d'autres hypothèses, et font apparaître plusieurs éléments nouveaux. D'une part, nous n'avons pas observé parmi les verbes de base des $\mathrm{N}$-age de surreprésentation des verbes transitifs, et le type aspectuel principalement sélectionné par les $\mathrm{N}$-age néologiques est celui des activités. Les effets observés dans les travaux existants sur la transitivité des bases et la tendance à privilégier la construction à partir de verbes d'accomplissement pourraient donc être contingents, et ne pas dépendre fondamentalement de l'opération de dérivation en -age. D’autre part, il est apparu que dans les formations néologiques, la suffixation en -age se caractérisait par sa transparence syntaxique et sémantique, les N-age construits décrivant généralement le même type d'éventualité que leur base, tout en préservant leurs propriétés aspectuelles et argumentales. On peut donc penser que les décalages aspectuels observés dans le lexique établi entre verbes et $\mathrm{N}$-age dérivés sont dus au processus de lexicalisation, et non à la construction morphologique en soi.

L'étude fournit en outre quelques informations sur la polysémie des N-age néologiques. Les configurations polysémiques non arbitraires, i.e. associées à une même acception du verbe de base, répondent très souvent à un même schéma sémantique, celui de l'association entre une action et son résultat. Les éléments rassemblés ne montrent pas que les acceptions résultatives soient dérivées de verbes, mais vont plutôt dans le sens d'une construction par métonymie à partir d'une acception nominale d'action. De fait, les $\mathrm{N}$-age néologiques qui ne dénotent pas des éventualités ne sont jamais des acceptions nominales uniques. Au plan sémantique, le meilleur prédicteur d'une polysémie 'action' / 'résultat' est la coprésence d'une culmination et d'un objet affecté dans la structure de l'acception verbale ou nominale à l'origine du sens résultatif.

Enfin, il apparaît dans les données analysées que la formation de néologismes en -age peut être diversement motivée, et notamment que la création en surabondance est tout à fait possible, même si les noms créés ne sont pas tous destinés à se lexicaliser. Cette surabondance témoigne in fine de la capacité de régénération lexicale inhérente au système dérivationnel. 


\section{RÉFÉRENCES}

Apresjan, J. (1974). Regular polysemy. Linguistics, 42: 5-32.

Aronoff, M. (2016). Competition and the lexicon. In: A. Elia, C. Iacobini et M. Voghera (dir.), Livelli di Analisi e fenomeni di interfaccia. Rome: Bulzoni, pp. 39-52.

Asher, N. (1993). Reference to Abstract Objects in Discourse. Dordrecht: Springer.

Bach, E. (1986). The algebra of events. Linguistics and Philosophy, 9: 5-16.

Balvet, A., Barque, L., Condette, M.-H., Haas, P., Huyghe, R., Marín, R. et Merlo, A. (2011). La ressource Nomage : Confronter les attentes théoriques aux observations du comportement linguistique des nominalisations en corpus. TAL, 52.3: 129-152.

Barque, L. (2008). Description et formalisation de la polysémie régulière en français. Thèse de doctorat, Université Paris Diderot.

Barque, L., Candito, M. et Huyghe, R. (2019). La classification des verbes réfléchis à l'épreuve d'une annotation en corpus. Langages, 216: 121-137.

Berrendonner, A. et Clavier V. (1997). Examen d'une série morphologique dite "improductive » en français : les noms dénominaux en -age. Silexicales, 1: 35-44.

Bertinetto, P. M. et Squartini, M. (1995). An attempt at defining the class of 'gradual completion' verbs. In: P. M. Bertinetto, V. Bianchi, J. Higginbotham et M. Squartini (dir.), Temporal Reference Aspect and Actionality 1. Semantics and Syntactic Perspectives. Torino: Rosenberg et Sellier, pp. 11-26.

Boons, J.-P., Guillet, A. et Leclère, C. (1976). La structure des phrases simples en français : constructions intransitives. Genève: Droz.

Bréal, M. (1897) [2005]. Essai de sémantique. Limoges : Lambert-Lucas.

Copestake, A. et Briscoe, T. (1995). Semi-productive Polysemy and Sense Extension. Journal of Semantics, 12.1: $15-67$.

Corbin, D. (1987). Morphologie dérivationnelle et structuration du lexique. Tübingen: Niemeyer.

Creissels, D. (2007). Réflexivisation, transitivité et agent affecté. In: A. Rousseau, D. Bottineau et D. Roulland (dir.), L'énoncé réfléchi. Rennes: Presses Universitaires de Rennes, pp. 83-106.

Dal, G., Hathout, N., Lignon, S., Namer, F. et Tanguy, L. (2018). Toile versus dictionnaires : Les nominalisations du français en -age et en -ment. In: F. Neveu, R. Harmegnies, L. Hriba et S. Prévost (dir.), Congrès Mondial de Linguistique Française 2018. Paris : Institut de Linguistique Française.

Dal, G. et Namer, F. (2016). À propos des occasionnalismes. In: F. Neveu, R. Harmegnies, L. Hriba et S. Prévost (dir.), Congrès Mondial de Linguistique Française 2016. Paris : Institut de Linguistique Française, pp. 1-18.

Dowty, D. (1979). Word Meaning and Montague Grammar. Dordrecht : Kluwer.

Dubois, J. (1962). Étude sur la dérivation suffixale en français moderne et contemporain. Paris: Larousse.

Dubois, J. et Dubois-Charlier, F. (1997). Les verbes français. Paris: Larousse-Bordas.

Ferret, K., Soare, E. et Villoing, F. (2010). Les noms d'événement en -age et en -ée : une différenciation fondée sur l'aspect grammatical. In: F. Neveu, V. Muni Toke, T. Klingler, J. Durand, L. Mondada et S. Prévost (dir.), Congrès Mondial de Linguistique Française 2010. Paris : Institut de Linguistique Française, pp. 945-968.

Ferret, K. et Villoing, F. (2012). L'aspect grammatical dans les déverbaux en -age et -ée. Lexique, 20: 73-127.

Ferret, K. et Villoing, F. (2015). French N-age instrumentals: Semantic properties of the base verb. Morphology, 25: 473-496.

Filip, H. (1999). Aspect, Eventuality Types and Nominal Reference. New York et London: Garland Publishing.

Flaux, N. et Van de Velde, D. (2000). Les noms en français. Esquisse de classement. Paris: Ophrys.

Fradin, B. (2012). Les nominalisations et la lecture 'moyen'. Lexique, 20: 125-152.

Fradin, B. (2016). L'interprétation des nominalisations en N-age et N-ment en français. In: E. Buchi, J.-P. Chauveau et J.-M. Pierrelm (dir.), Actes du XXVIIe Congrès International de Linguistique et de Philologie Romane. Strasbourg : Société de Linguistique Romane/Eliphi, pp. 53-66.

Fradin, B. (2019). Competition in derivation: what can we learn from french doublets in -age and -ment? In: F. Rainer, F. Gardani, W. U. Dressler et H. C. Luschützky (dir.), Competition in Inflection and Derivation. Cham: Springer, pp. 67-93. 
Fradin, B. et Kerleroux, F. (2003). Quelle base pour les procédés de la morphologie constructionnelle ? Silexicales, 3: 76-84.

Gardani, F., Rainer, F., et Luschützky, H. C. (2019). Competition in morphology: a historical outline. In: F. Rainer, F. Gardani, W. U. Dressler et H. C. Luschützky (dir.), Competition in Inflection and Derivation. Cham: Springer, pp. 3-36.

Godard, D. et Jayez, J. (1996). Types nominaux et anaphores : le cas des objets et des événements. Cahiers Chronos, 1: 41-58.

Grimshaw, J. (1990). Argument Structure. Cambridge: The MIT Press.

Haas, P., Huyghe, R. et Marín, R. (2008). Du verbe au nom : calques et décalages aspectuels. In: J. Durand, B. Habert et B. Laks (dir.), Congrès Mondial de Linguistique Française. CMLF 2008. Paris : Institut de Linguistique Française, pp. 2051-2065.

Hay, J., Kennedy, C. et Levin, B. (1999). Scalar structure underlies telicity in 'degree achievements'. In: T. Mathews et D. Strolovitch (dir.), SALT 9. Ithaca: CLC Publications, pp. 127-144.

Huyghe, R. (2015a). Les nominalisations « d'achèvement graduel » en français. Le Français Moderne, 83: $18-33$.

Huyghe, R. (2015b). Les typologies nominales : présentation. Langue Française, 185: 5-27.

Jacquey, E. (2006). Un cas de " polysémie logique » : modélisation de noms d'action en français ambigus entre processus et artefact. TAL, 47.1: 137-166.

Kelling, C. (2001). Agentivity and suffix selection. In: M. Butt, et T. Holloway King (dir.), Proceedings of the LFG01 Conference. Stanford: CSLI Publications, pp. 147-162.

Kleiber, G., Benninger, C., Biermann Fischer, M., Gerhard-Krait, F., Lammert, M., Theissen, A. et Vassiliadou, H. (2012). Typologie des noms : le critère de se trouver + SP locatif. Scolia, 26: $105-129$.

Lüdtke, J. (1978). Prädikative Nominalisierungen mit Suffixen im Französischen, Katalanischen und Spanischen. Tubingen: Narr.

Martin, F. (2010). The semantics of eventive suffixes in French. In: A. Alexiadou et M. Rathert (dir.), The Semantics of Nominalizations across Languages and Frameworks. Berlin: Mouton de Gruyter, pp. 109-140.

Mel'čuk, I. (1993). Cours de morphologie générale, vol. 1. Montréal: Presses de l'Université de Montréal.

Melloni, C. (2011). Event and Result Nominals. A Morpho-semantic Approach. Berne: Peter Lang.

Missud, A. (2019). Modélisation quantitative de la rivalité entre -age et la conversion de verbe à nom. Mémoire de Master, Université Paris Nanterre.

Namer, F. (2009). Morphologie, lexique et traitement automatique des langues. Paris: Hermès Lavoisier.

Namer, F. et Villoing, F. (2008). Interpréter les noms déverbaux : quelle relation avec la structure argumentale du verbe de base ? Le cas des noms en -oir du français. In: J. Durand, B. Habert et B. Laks (dir.), Congrès Mondial de Linguistique Française. CMLF 2008. Paris : Institut de Linguistique Française, pp. 1551-1569.

New, B., Pallier, C., Ferrand, L. et Matos, R. (2001). Une base de données lexicales du français contemporain sur internet : Lexique. L'année Psychologique, 101: 447-462.

Plag, I. (1999). Morphological Productivity: Structural Constraints in English Derivation. Berlin: Mouton de Gruyter.

Plag, I., Andreou, M. et Kawaletz, L. (2018). A frame-semantic approach to polysemy in affixation. In O. Bonami, G. Boyé, G. Dal, H. Giraudo. et F. Namer (dir.), The lexeme in descriptive and theoretical morphology. Berlin: Language Science Press, pp. 467-486.

Pustejovsky, J. (1995). The Generative Lexicon. Cambridge Mass.: The MIT Press.

Rothstein, S. (2004). Structuring Events: A Study in the Semantics of Lexical Aspect. Oxford: Blackwell.

Sagot, B. (2010). The Lefff, a freely available and large-coverage morphological and syntactic lexicon for French. In: N. Calzolari, K. Choukri, B. Maegaard, J. Mariani, J. Odijk, S. Piperidis, M. Rosner et D. Tapias (dir.), Proceedings of the 7th international conference on Language Resources and Evaluation (LREC 2010). Valetta: ELRA.

Thornton, A. M. (2012). Reduction and maintenance of overabundance. A case study on Italian verb paradigms. Word Structure, 5: 183-207. 
Uth, M. (2010). The rivalry of the French nominalization suffixes -age and -ment from a diachronic perspective. In: A. Alexiadou et M. Rathert (dir.), The Syntax of Nominalizations across Languages and Frameworks, (eds), Berlin: De Gruyter, pp. 215-244.

Verkuyl, H. J. (1993). A Theory of Aspectuality. Cambridge: Cambridge University Press.

Wauquier, M., Fabre, C. et Hathout, N. (2018). Différenciation sémantique de dérivés morphologiques à l'aide de critères distributionnels. In: F. Neveu, R. Harmegnies, L. Hriba et S. Prévost (dir.), Congrès Mondial de Linguistique Française 2018. Paris : Institut de Linguistique Française.

Cite this article: Huyghe R and Lombard A (2022). Les néologismes en -age en français contemporain : héritage verbal et polysémie. Journal of French Language Studies 32, 25-47. https://doi.org/10.1017/ S0959269520000320 